\title{
Profil Epidemiologique De La Leishmaniose Cutanee Dans La Region Du Gharb- Maroc De 2006 À 2014
}

\author{
Aziz El Aasri \\ Alaoui Zakaria \\ Khadija EI Kharrim \\ Driss Belghyti
}

Laboratoire Biotechnologie et Environnement (LABEQ), Equipe de Parasitologie et Environnement, Département de Biologie, Faculté des

Sciences, Université, Ibn Tofaïl, Kénitra, Maroc

\section{Yassine Aqachmar}

Chef de service d'infrastructure des actions ambulatoires provincial,

Hôpital Provincial de Sidi Kacem, Maroc

\section{Mohamed Hadji}

Laboratoire D’épidémiologie Provinciale, Délégation De Santé, Sidi Slimane

doi: 10.19044/esj.2016.v12n3p243 URL:http://dx.doi.org/10.19044/esj.2016.v12n3p243

\begin{abstract}
Between 2006 and 2014, 439 cases of cutaneous leishmaniasis were recorded in the region of Gharb Chrarda Beni Hssen in the north-west of Morocco. With an annual incidence of 49.1 cases per year and a sex-ratio ( $\mathrm{M} / \mathrm{F}$ ) of 0.71 . The disease has affected all age groups. The most stricken population is children and young people between the ages of 6 months and 30 years with more than $60.26 \%$ of cases. Therefore, Cutaneous leishmaniasis almost hits the rural and urban areas but with an uneven impact. It is higher in rural areas where they are registered with a percentage of $56.7 \%$ of cases in contrast to a percentage of $43.3 \%$ in urban areas.
\end{abstract}

Keywords: Epidemiology, Parasitology, Cutaneous Leishmaniasis, Kenitra, Morocco

\section{Resume}

Entre 2006 et 2014, 439 cas de leishmaniose cutanée ont été enregistrés dans la région du Gharb Chrarda Beni Hssen au nord-ouest du Maroc. Avec une incidence annuelle de 49,1 cas par an et un sexe-ratio (H/F) de 0,71. La maladie a touché toutes les tranches d'âges. Les enfants et les jeunes dont l'âge compris entre 6 mois et 30 ans constituent la population 
la plus frappée avec plus de 60,26\% des cas. Ainsi, La leishmaniose cutanée frappe pratiquement le milieu rural et urbain avec cependant une incidence inégale. Elle est plus importante dans les secteurs ruraux où-ils sont enregistrés $56,7 \%$ des cas contre 43,3\% dans les secteurs urbains.

Mots Clés: Épidémiologie, Parasitologie, Leishmaniose cutanée, Kénitra, Maroc

\section{Introduction}

La leishmaniose cutanée (LC) est la forme la plus commune des leishmanioses. C'est une infection cutanée provoquée par un parasite unicellulaire du genre Leishmania qui est transmis par des piqures de phlébotome (Diptera : Psychodidae ) (Guernaoui, 2006). Elle représente un problème de santé publique tant au niveau mondial avec une incidence annuelle estimée à 1500000 cas qu'à l'échelle du Maroc où elle sévit dans toutes les régions du royaume de manière endémo-épidémique (Desjeux, 1996 ; OMS, 2010 ; Zouirech et al.,...).

L'objectif de cette étude rétrospective et prospective est d'analyser la distribution spatiale et temporelle des leishmanioses cutanée a fin d'évaluer le profil épidémiologique de ces parasitoses dans la région du Gharb Chrarda Beni Hssen (GCBH) à nord-ouest du Maroc entre 2006 et 2014.

\section{Milieu d'étude}

Regroupant 3 provinces (Kénitra, Sidi Kacem et Sidi Slimane), 10 communes urbaines et 53 communes rurales, la région du Gharb-ChrardaBeni Hssen se situe dans la partie Nord-Ouest du Royaume. Elle est limitée à l'Ouest par l'Océan Atlantique, au Nord par la région de Tanger-Tétouan, à l'Est par les deux régions de Taza-Al Hoceima-Taounate et de FèsBoulemane, et au Sud par les régions de Meknès-Tafilalet et de Rabat-SaléZemmour-Zaër. Elle s'étend sur environ $7900 \mathrm{~km}^{2}$, soit près de 1,08\% de la superficie du Maroc. Sa Population est de 1859540 habitants, soit $6.22 \%$ de la population nationale. La densité moyenne est de $211 \mathrm{hab} / \mathrm{km}^{2} .5$ fois plus élevée que la densité nationale ( $\left.40 \mathrm{hab} / \mathrm{Km}^{2}\right)$.

\section{Matériels et méthodes}

La méthode utilisée pour diagnostiquer les leishmanioses cutanées dans la région ouest marocaine est l'examen parasitologique direct qui consiste à l'identification microscopique du corps leishmania (amastigotes) après coloration au MAYGRÜNWALD GIEMSA (MGG) (Rhajaoui, 2009). En effet, toutes les préparations microscopiques (frottis) qui s'effectuent par des personnels infirmiers au niveau des différentes circonscriptions sanitaires appartenant a la délégation de Sidi Kacem seront envoyées au laboratoire 
d'épidémiologie et d'hygiène du milieu «L.E.H.M » du service d'infrastructure des actions ambulatoires provincial «S.I.A.A.P » de Sidi Kacem pour la coloration et l'identification. Les delegations de Sidi Slimane et Kénitra adressent leurs frottis au laboratoire de l'institut national d’hygiène « INH » à Rabat.

\section{Résultats}

\section{Répartition temporelle}

Sur l'ensemble des fiches et des registres exploités, le sexe a été précisé chez 468 patients (le sexe n’a pas été mentionné sur 10 fiches). La répartition temporelle des cas selon le sexe est représentée dans le tableau 1.

Tableau 1 : Distribution des fréquences de LC en fonction du sexe et des années.

\begin{tabular}{cccccccccccc}
\hline \multirow{2}{*}{ Sexe } & \multicolumn{10}{c}{ Année } \\
\cline { 2 - 14 } & 2006 & 2007 & 2008 & 2009 & 2010 & 2011 & 2012 & 2013 & 2014 & Total & $\%$ \\
\hline Masculin & 8 & 13 & 14 & 20 & 30 & 34 & 30 & 26 & 35 & 210 & 44,88 \\
\hline Féminin & 17 & 21 & 22 & 32 & 34 & 39 & 28 & 36 & 29 & 258 & 55,12 \\
\hline Total & 25 & 34 & 36 & 52 & 64 & 73 & 58 & 62 & 64 & 468 & 100 \\
\hline
\end{tabular}

Avec une moyenne de 49,1 cas par an, le nombre total des cas de leishmaniose cutanée enregistré dans la région du GCBH présente une évolution en oscillation croissante entre 2006 et 2014. Le minimum de cas a été enregistré en 2006 avec 25 cas et le maximum en 2011 avec 73 cas. Ainsi, la leishmaniose cutanée atteint les deux sexes avec une prédominance féminine dont le pourcentage était de $58,6 \%$ contre $41,4 \%$ pour le sexe masculin et un sexe ratio (H/F) de 0,71 .

\section{Répartition selon la nature du milieu}

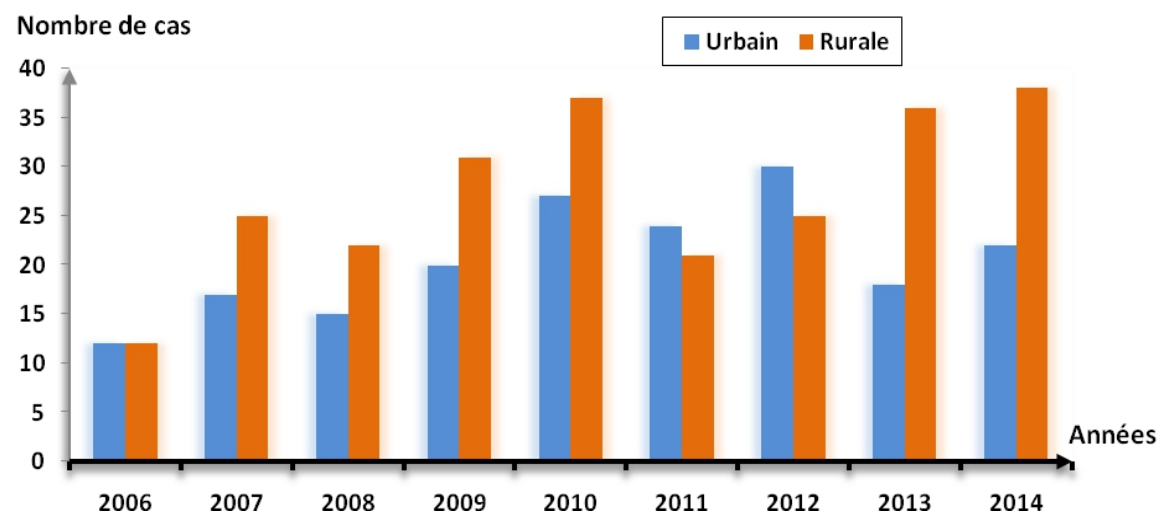

Figure 1: Répartition des cas de LC selon le type de milieu 
Dans la région du GCBH, La leishmaniose cutanée frappe pratiquement le milieu rural et urbain avec cependant une incidence inégale. En effet, elle est plus importante dans les secteurs ruraux où sont enregistrés $56,7 \%$ des cas contre $43,3 \%$ dans les secteurs urbains (Figure 1). Cette légère prédominance des cas ruraux est observée durant toutes les années à l'exception de 2006, 2011 et 2012.

\section{Répartition par Tranches d'âge}

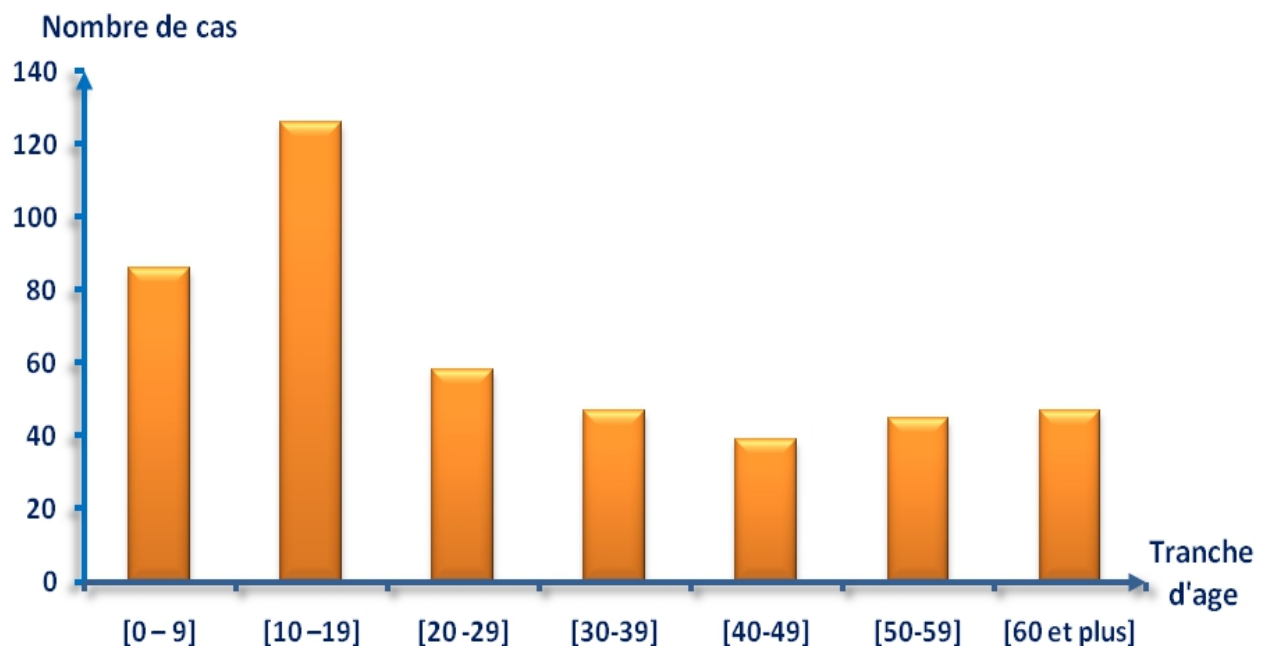

Figure 2: Répartition des LC selon les tranches d'âge

Entre 2006 et 2014, toutes les tranches d'âge sont touchées par la leishmaniose cutanée dans la partie nord-ouest marocaine, mais avec des proportions inégales. En effet, la classe la plus touchée est celle des jeunes et enfants dont l'âge est compris entre 10 et 19 ans avec 28,1\% alors que la moine atteinte se situe entre 40 et 49 ans (Figure 2). 


\section{Cartographie de LC au Nord-Ouest du Maroc}

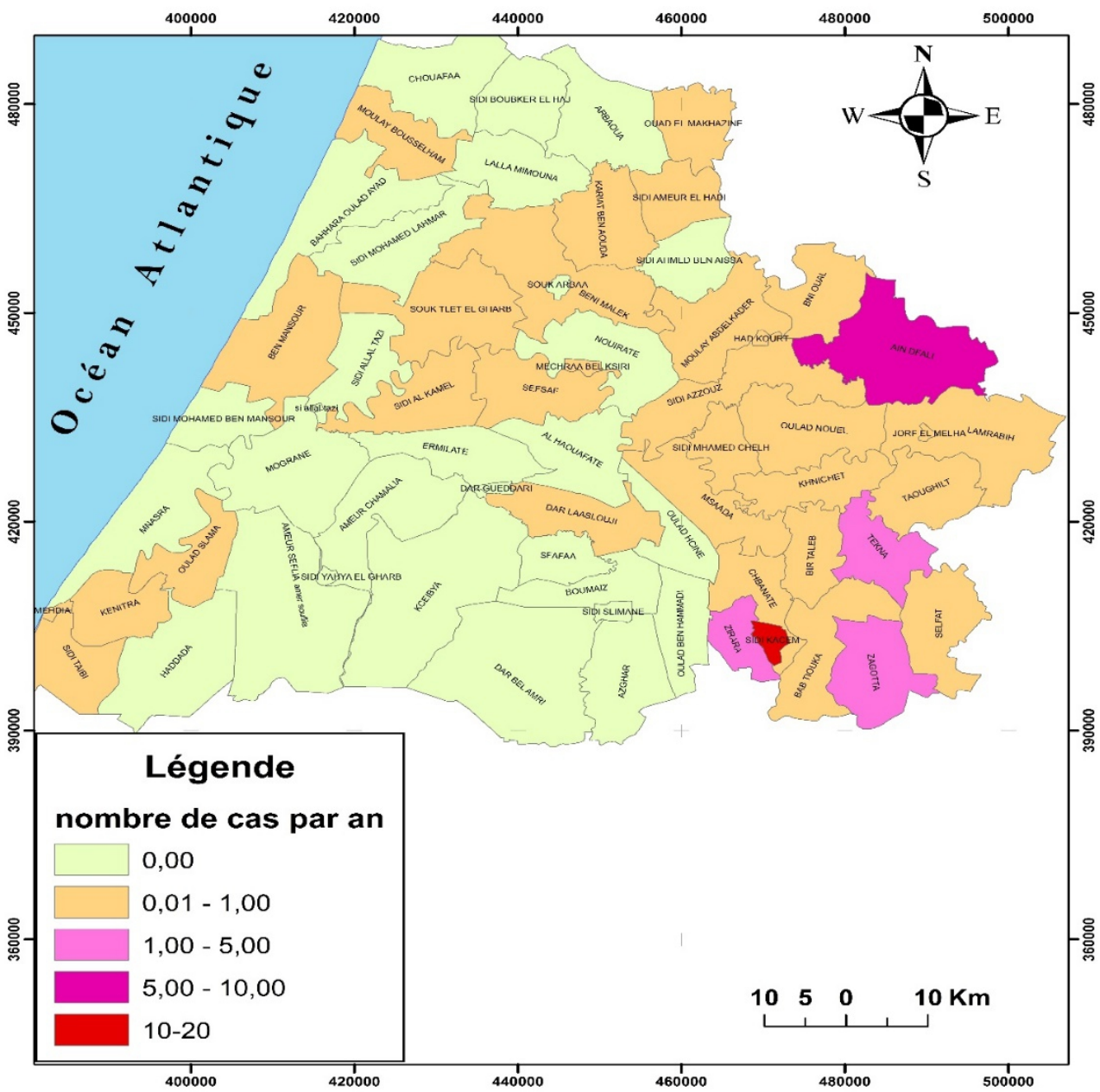

Figure 3: Répartition des cas de LC par commune à l'ouest du Maroc

Les résultats obtenus représentés sur la carte (Figure 3), montrent une forte concentration des cas dans les communes occupant la partie est de la région, alors que celles du centre et de l’ouest ont été moins frappées. La commune la plus atteinte était celle urbaine de Sidi Kacem avec une moyenne de 15,66 cas par an. La commune rurale d'Ain Dfali, avec la moyenne de 7,33 cas par an, occupe la deuxième place. La moyenne annuelle des cas comprise entre 1 et 5 cas a été enregistrée au niveau de trois communes rurales, il s'agit de Zirara, Zeggota et Tekna avec successivement 3,$22 ; 1,67$ et 1,11 cas par an. Les communes où la moyenne annuelle des cas par an est supérieure à 1 appartiennent toutes à la province de Sidi Kacem. Le nombre réduit de cas (inferieur à 1) était enregistré au sein de 31 parmi les 53 communes constituant la région du GCBH. Nous remarquons aussi 
une absence de cas dans 13 communes occupant surtout le centre et l'ouest de notre zone d'étude.

\section{Discussion}

Les résultats obtenus durant la période allant du mois de janvier 2006 au mois de décembre 2014, montrent que la répartition spatiale de LC au Nord-Ouest du Maroc n'est pas homogène. En effet, selon ces résultats, une forte concentration des cas fût enregistrée dans la partie Est où sont situées également les communes les plus atteintes, alors que le centre et l'ouest de la région étaient moins frappés (Figure 3). La distribution par prefecture montre que parmi l'ensemble des cas retrouvés, 446 sont originaires de la province de Sidi Kacem, 24 de kénitra et 8 de Sidi Slimane. Cette répartition inégale peut être due à l'action des facteurs environnementaux, surtout ceux liés au climat et à la géographie. En effet, les préfectures les moines frappées (Kénitra et Sidi Slimane) occupent l'ouest et le centre de la région où l'effet océanique existe et l'altitude faible, alors que la province la plus touchée de Sidi Kacem, occupe l'est de la région et se trouve donc loin de l'océan atlantique et sur une altitude plus élevée que celle de Kénitra et Sidi Slimane. Il apparait donc qu'en plus de l'altitude dont l'effet est déjà confirmé sur la répartition spatiale des LC et leurs vecteurs (Abonnenc, 1972), l'effet océanique et la continentalité semblent à leurs tours influencer la distribution géographique de ces parasitoses.

Concernant la répartition temporelle durant les 9 années concernées par notre étude, nous avons trouvé une moyenne de 53,11 cas de LC par an. L'évolution de la fréquence des cas selon les années était en oscillation croissante avec un maximum de cas en 2011 (73 cas). Cette augmentation s'expliquerait, d'une part par l'amélioration de la déclaration et de la prise en charge des malades et d'autre part par la recrudescence effective du nombre de cas, favorisée par les modifications écologiques du milieu liées, aux changements climatiques, au développement agricole et l'exploitation de nouvelles ressources hydrauliques (installation des canaux d'irrigation, puits artésiens,...etc) et à l'urbanisation anarchique (bidonvilles et quartiers insalubres). Tous ces facteurs ont aboutit à la création des microclimats humides favorables aux développement des phlébotomes et donc aux cycles des leishmanioses (Killick-Kendrick, 1999 ; Bousaa, 2008; Zouirech et al.,... ; OMS, 2010).

Pour la sex-ratio, nous avons remarqué que la prédominance était féminine avec 258 cas soit $44,88 \%$ contre 210 cas de sexe masculin soit $55,12 \%$ de cas. Ce résultat est semblable à celui rapporté par Chiheb et ces collaborateurs au nord du Maroc en 1999 (Chiheb et al., 1999; Elassri, 2006). Il pourrait être expliqué d'une part, par le fait que les femmes sont beaucoup plus impliquées que les hommes dans les activités favorisant la 
maladie, ou bien par le fait que les hommes, en raison de leur occupation ou par négligence ne consultent que rarement ou seulement en cas de complications ce qui est en faveur d'une sous déclaration masculine. En 2003, la predominance féminine a été également rapportée en Tunisie (Masmoudi et al., 2005) et au Burkina-Faso (Traoré et al., 2011), alors que le sexe ratio était très en faveur du sexe masculin au Mali (Famakan, 2005) et en Guyane (El-safi, 1991.

Parmi les cas de LC retrouvés durant cette étude, 56,7\% appartiennent au milieu rural contre $43,3 \%$ originaires du milieu urbain ce qui confirme le caractère rural de cette parasitose (OMS, 2014). Ainsi, dans le milieu urbain, la plus part des cas ont été signalés au niveau des quartiers insalubres et périphériques ce qui confirme le rôle que joue l'urbanisation anarchique dans la propagation de cette parasitose vers les centres urbains (Boussaa, 2008).

Notre étude a montré que toutes les tranches âges étaient touchées par la LC, les enfants et les jeunes dont l'âge est compris entre 6 mois et 30 ans constituent la population la plus frappée avec plus de 60,26\% des cas. L’âge moyen des patients atteints était de 24,7 ans. Ce résultat est très proche de celui obtenu par Chiheb en 1999 au nord du Maroc qui a rapporté 24,6 ans comme moyenne d’âge (Chiheb et al., 1999). En 2003, Famakan a obtenu une moyenne d’âge de 24 ans au Mali, alors qu'en Tunisie Kharfi et ces collaborateurs ont trouvé une moyenne d’âge plus élevée (42,4 ans) (Kharfi et al., 2003).

\section{Conclusion}

$\mathrm{Au}$ terme de cette étude rétrospective, nous concluons que la leishmaniose cutanée constitue un problème de santé publique au Nord-ouest du Maroc où elle évoluait selon le mode hypo-endémique croissant, et peut donc devenir endémique au futur surtout dans la province de Sidi Kacem qui est limitrophe de la zone d'endémie d’Ouazzane. Aussi, il est important de noter que les données recueillies entre 2006 et 2014 ne sont pas exhaustives et ne reflètent pas la situation exacte de cette parasitose puisque la plupart des patients atteints, attendent la guérison spontanée et ne consultent qu'en cas de complications, ce qui est en faveur d’une sous déclaration.

\section{References:}

Abonnenc E., 1972 : Les phlébotomes de la région éthiopienne (Diptera: Phlebotomidae). Mémoire de l'ORSTOM. 55, 1-289.

Boussaa S., 2008: Epidémiologie des leishmanioses dans la région de Marrakech, Maroc: effet de l'urbanisation sur la répartition spatio-temporelle des Phlébotomes et caractérisation moléculaire de leurs populations. Thèse 
de doctorat en sciences de la vie et de la santé, Université Louis Pasteur Strasbourg I, 207p.

Chiheb S., Guessous-Idrissi N., Hamdani A., Riyad M., Hamdani S. \& Krimech A. (1999). Cutaneous leishmaniasis due to Leishmania tropica: clinical features in a new focus in northern Morocco. Ann Dermatol Vénérol, 126 (5): 419-425.

Desjeux P. (1996). Leishmaniasis: public health aspects and control. Clin. Dermatol. 14, 417- 423.

El Safi S.H., Peters W., El-Toam B., El-Kadarow A. and Evans, D.A. (1991). Studies on the leishmaniases in the Sudan: 2. Clinical and parasitological studies on cutaneous leishmaniasis. Transactions of the Royal Society of tropical Medicine and Hygiene. 85, 457-464.(UK).

Famakan K., 2005 : Thèse de pharmacie. La leishmaniose cutanée chez les patients reçus à l'unité biologie du CNAM de janvier 2002 à Octobre 2004, Bamako.

Guernaoui S., 2006: Les leishmanioses dans les zones arides et semi-arides du sud-ouest marocain. Ecologie, épidémiologie, modélisation et aide à la décision pour la lutte antivectorielle. Thèse de Doctorat. Faculté des Sciences, Semlalia, Marrakech. 166p.

Kharfi M., Fazaa B., Chaker E., Kamou M.R., 2003: Localization muqueuse de la leishmaniose en Tunisie : 5 observations. Annales de Dermatologie et de vénéréologie. vol.96 (5 ) : 383 - 388.

Killick-Kendrick R. \& Killick-Kendrick M. (1999). Biology of sand fly vectors of Mediterranean canine leishmaniasis. In : Canine Leishmaniasis : an up date. Proceedings of a canine Leishmaniasis Forum, Barcelona, 28-31. Masmoudi A. et Kitar A., 2005: La leishmaniose cutanée de la face dans la région de Gafsa, Tunisie. Clinique : 1-6.

OMS. 2010: Rapport de la réunion du comité OMS d'experts de la lutte contre les leishmanioses. Genève . 22-26.

OMS. 2011 : Agir pour réduire l'impact mondial des maladies tropicales négligées. Rel Epidem Hebd. N0 13, 86 : 113-128.

Rhajaoui M., Nasereddin A., Fellah H., Azmik K., Amarir F., Al-Jawabreh A., Ereqat S., Planer J. \& Abdeen Z. (2007). New clinico-epidemiologic profile of cutaneous leishmaniasis, Morocco. Emerg Infect Dis. 13, 13581360.

Traore KS, Sawadogo NO, Traore A, 2001: Etude préliminaire de la leishmaniose cutanée dans la ville de Ouagadougou de 1996- 1998 . Bulletin de la société de pathologie exotique. Vol. 24(1): 52-55.

Zouirech M., Belghyti D., El-Kohli M. \& Faraj C. (2013). Entomological investigation of an emerging leishmaniasis focus in Azilal province, Morocco. Pakistan Entomologist 35, p. 11-15. 